Rapid Communications

\title{
Noncontacting optostriction driven anisotropic and inhomogeneous strain in two-dimensional materials
}

\author{
Jian Zhou $\odot,{ }^{1, *}$ Sheng Mao $\odot,{ }^{2,3}$ and Shunhong Zhang $\odot^{4}$ \\ ${ }^{1}$ Center for Advancing Materials Performance from the Nanoscale, State Key Laboratory for Mechanical Behavior of Materials, Xi'an \\ Jiaotong University, Xi'an 710049, China \\ ${ }^{2}$ Department of Mechanics and Engineering Science, BIC-ESAT, College of Engineering, Peking University, Beijing 100871, China \\ ${ }^{3}$ Department of Mechanical and Aerospace Engineering, Princeton University, Princeton, New Jersey 08544, USA \\ ${ }^{4}$ International Center for Quantum Design of Functional Materials (ICQD), Hefei National Laboratory for Physical Sciences at Microscale, \\ University of Science and Technology of China, Hefei, Anhui 230026, China
}

(Received 27 October 2019; accepted 29 April 2020; published 15 June 2020)

\begin{abstract}
Strain engineering has been well developed and widely used to manipulate properties of materials. For twodimensional materials, via nanoindentation technique or depositing the materials onto flexible substrates, one usually triggers tensile strains. It would be intriguing to develop strategies to generate strains via noncontacting schemes, such as an optical field, to eliminate lattice damage and additional interactions. Here we theoretically and computationally illustrate an optomechanical approach (referred to as optostriction), which could induce intrinsic strains in materials. Taking the well-studied transition metal dichalcogenide monolayers as examples, we predict a large in-plane optostriction with strong anisotropy, owing to their unique directional band transition strength. This optically driven strain method can avoid direct and invasive mechanical contacts with materials, which is easily accessible and guarantees the reversibility of materials. Rather than nanoindentation technique and other similar methods, this optomechanical strain can be either tensile or compressive. Owing to its intrinsicality, compressive strains are robust without suffering Euler's instability. In-plane inhomogeneous strains can be easily achieved via illuminating a Gaussian beam onto the material, adding an interesting approach to realize and measure in-plane optoflexoelectricity. Unlike conventional electric field inducing strains in piezoelectrics, no symmetry constraints are required.
\end{abstract}

DOI: 10.1103/PhysRevResearch.2.022059

Controlling and manipulating structures and properties of materials via noncontacting schemes, such as light illumination, have been a long-standing promising approach. One interesting phenomenon pertinent to this technique is optomechanical coupling which efficiently converts photonic energy into mechanically elastic or plastic deformation energy involving a nonzero stress/strain. The stress/strain state of a solid has profound effects on its thermodynamic stability and physical and chemical properties, which can be further employed in a wide range of perspective applications and discoveries [1-5]. As the growing demand of miniaturizing device sizes, interactions between light and nanoscale twodimensional (2D) materials have been receiving considerable attentions. With an ultrahigh surface-to-volume ratio, 2D materials are almost "all surface" geometrically, so that optical manipulation with focused laser is easily accessible with high efficiency.

\footnotetext{
*jianzhou@xjtu.edu.cn

Published by the American Physical Society under the terms of the Creative Commons Attribution 4.0 International license. Further distribution of this work must maintain attribution to the author(s) and the published article's title, journal citation, and DOI.
}

Many 2D materials hitherto discovered possess in-plane anisotropic electronic, mechanical, thermal, or optical behaviors [6-14]. In-plane anisotropy provides continuously tunable properties of them, yielding interesting applications under different circumstances. The study and understanding of anisotropic optomechanics of 2D materials is still in its infancy, which hinders its potential usage.

Strain engineering in 2D materials is a well-developed technique to manipulate its properties. Unfortunately, one can only apply in-plane tensile strains to 2D (or 1D) materials, because even a small amount of compressive strain induces buckling instability. Hence, it is interesting if one can trigger compressive strain intrinsically in materials with reduced dimensionality, which would largely extend their stabilities and applications. Another interesting problem is how to generate spatially in-plane inhomogeneous strain field, which aids to produce promising properties, such as in-plane flexoelectric polarizations. Note that currently most flexoelectricity occurs in the out-of-plane direction via bending 2D materials.

In this Rapid Communication we theoretically and computationally illustrate an optomechanical response, which we refer to as "optostriction." Applying fundamental thermodynamic theory, we show that intermediate intensity laser shining onto the material makes it undergo an elastic deformation to release excessive stress and keep its Gibbs free energy profile. We use a family of well-studied topological insulators, 
group-VI transition metal dichalcogenide (TMD) monolayers in their $1 T^{\prime}$ phase, which is centrosymmetric with no spontaneous polarization, to illustrate our theory. This phase has been fabricated by multiple approaches [15-21], and its topological nature has been verified in various experiments [22-26]. In the $1 T^{\prime}$-TMD monolayers, band inversion occurs around the $\Gamma$ point of the Brillouin zone (BZ), making it belong to $Z_{2}$ topological insulators [27]. The direct band gap of $1 T^{\prime}$-TMD monolayers lies along the $\Gamma \rightarrow Y$ path. The band symmetry character makes it optically anisotropic in the $x y$ plane. With these, even though they are almost isotropic mechanically, our density functional theory (DFT) calculations [28-35] show that they possess large in-plane anisotropic optostriction. Interestingly, we predict that large intrinsic compressive strain can be generated under an intermediate intensity laser illumination. By using a focused inhomogeneous laser (e.g., Gaussian profile beam), in-plane strain gradient can be easily realized, in addition to conventional out-of-plane strain gradient realized by mechanical bending.

We first perform thermodynamic analysis. We consider optical frequency below the allowed optical transition band gap, thus only optical electric field effect is considered. Under an (linearly polarized) optical electric field $\mathcal{E}\left(\omega_{0}, t\right)=\mathbf{E} \times$ $e^{-i \omega_{0} t}$, the internal energy density of a material increases as $d u=\operatorname{Re}\left(\mathcal{E} \cdot d \mathbf{D}^{*}\right)$ (isothermic process). Here $\mathbf{D}$ is electric displacement, which contains two parts, i.e., the static spontaneous $\mathbf{D}_{0}$ and electric field induced $\mathbf{D}_{\text {ind }}$. When we use $\mathcal{E}\left(\omega_{0}, t\right)$ as natural variable (corresponding to closed-circuit boundary condition, see Refs. [36,37]), under Legendre transformation, its effect can be taken into account by including an additional term in the Gibbs free energy,

$$
d \mathfrak{G}=\operatorname{Re}\left[-\mathbf{D}_{0}^{*} \cdot d \mathcal{E}\left(\omega_{0}, t\right)-\mathbf{D}_{\text {ind }}^{*} \cdot d \mathcal{E}\left(\omega_{0}, t\right)\right] .
$$

By integrating it and noting the linear constitutive relationship $\mathbf{D}_{\text {ind }}=\varepsilon_{0} \overleftrightarrow{\boldsymbol{\varepsilon}} \cdot \mathcal{E}\left(\varepsilon_{0}\right.$ is vacuum permittivity and $\overleftrightarrow{\boldsymbol{\varepsilon}}$ is second order complex relative permittivity tensor, $\left.\overleftrightarrow{\boldsymbol{\varepsilon}}=\overleftrightarrow{\boldsymbol{\varepsilon}^{\prime}}+i \times \stackrel{\leftrightarrow}{\boldsymbol{\varepsilon}^{\prime \prime}}\right)$,

$$
\begin{aligned}
\mathfrak{G}(\mathcal{E})= & \mathfrak{G}_{0}-\operatorname{Re}\left\langle\left[\mathbf{D}_{0}^{*} \cdot \mathcal{E}\left(\omega_{0}, t\right)\right]\right. \\
& \left.+\left[\boldsymbol{\varepsilon}_{0} \mathcal{E}^{*}\left(\omega_{0}, t\right) \cdot \overleftrightarrow{\boldsymbol{\varepsilon}}^{\prime}\left(\omega_{0}\right) \cdot \mathcal{E}\left(\omega_{0}, t\right) / 2\right]\right\rangle .
\end{aligned}
$$

When the selected frequency $\omega_{0}$ is larger than infraredactive phonon frequency (below $10 \mathrm{THz}$ ), the static displacement $\mathbf{D}_{0}$ is little influenced (see Ref. [28] for a detailed discussion). This off-resonating feature indicates that the $\mathbf{D}_{0}$ term in Eqs. (1) and (2) vanishes after taking time average $\langle\cdot\rangle$. Hence, the thermodynamic Gibbs free energy varies according to (Einstein summation convention),

$$
d \mathfrak{G}=-S_{i j} d X_{i j}-\left\langle D_{i}^{*} d \mathcal{E}_{i}\right\rangle=-S_{i j} d X_{i j}-\varepsilon_{0} \varepsilon_{i j}^{\prime}\left(\omega_{0}\right)\left\langle\mathcal{E}_{i}^{*} d \mathcal{E}_{j}\right\rangle .
$$

Here $S$ and $X$ are strain and stress tensors, respectively. Positive (negative) $S$ represents tensile (compressive) strain. Equation (3) demonstrates that optical electric field reciprocates with mechanical strain. Note that $\left\langle\mathcal{E}_{k}^{*} \mathcal{E}_{l}\right\rangle=E_{k} E_{l}$, we write the mechanical response to optical field explicitly,

$$
S_{i j}=\mathcal{M}_{i j k l}\left\langle\mathcal{E}_{k}^{*} \mathcal{E}_{l}\right\rangle=\mathcal{M}_{i j k l} E_{k} E_{l} .
$$

(a)

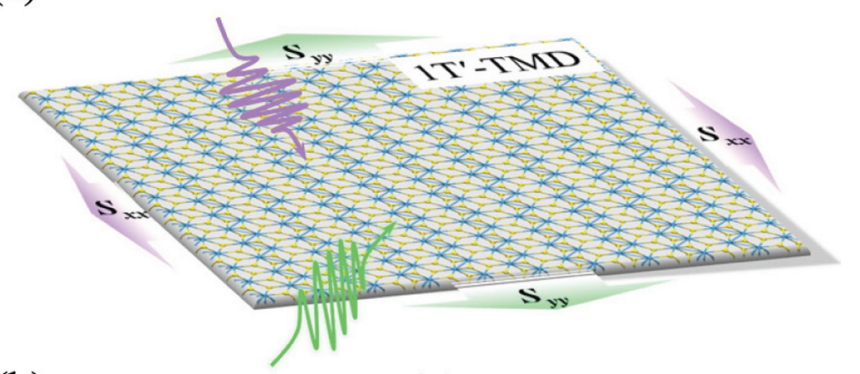

(b)

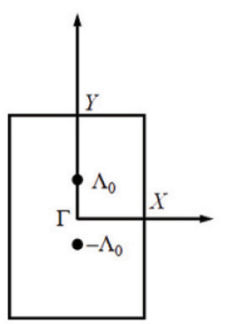

(c)

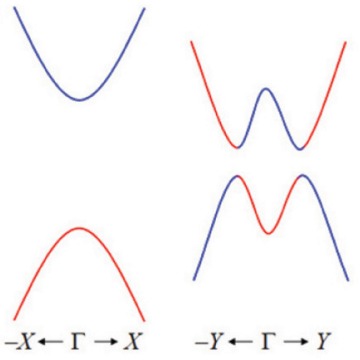

FIG. 1. (a) Geometric structure of $1 T^{\prime}$-TMD monolayer under linearly polarized light illumination. The structure would shrink or expand according to the dielectric function variation. (b) First Brillouin zone, with $\pm \Lambda_{0}$ denoting Dirac points without SOC. (c) Schematic band dispersion around $\Gamma$, with blue and red colors representing different orbitals, respectively.

It shows a quadratic relationship, rather than linear $E-S$ coupling in piezoelectrics. By taking derivatives,

$$
\begin{aligned}
\mathcal{M}_{i j k l} & =\frac{1}{2} \frac{\partial^{2} S_{i j}}{\partial E_{k} \partial E_{l}}=-\frac{1}{2} \frac{\partial^{3} \mathfrak{G}}{\partial X_{i j} \partial E_{k} \partial E_{l}} \\
& =\frac{1}{2} \frac{\partial^{2} D_{k}}{\partial X_{i j} \partial E_{l}}=\frac{1}{2} \frac{\varepsilon_{0} \partial \varepsilon_{k l}^{\prime}\left(\omega_{0}\right)}{\partial X_{i j}} .
\end{aligned}
$$

The coefficient $1 / 2$ is added to correct double counting in Einstein summation, and $\mathcal{M}$ is a Voigt symmetric tensor [38]. In contrast to the photoinduced mechanical deformation (photostriction [39-42] or quantum electronic stress $[43,44]$ ) where photons are absorbed by the material, here the optical incident energy is below direct transition band gap. Therefore, upon light illumination, only wavelike optical electric field effects are accounted, while the adsorption of photon (particle feature) can be ignored. This would significantly reduce the overheating problem from the non-radiative electron-hole recombination process.

We now illustrate this optostriction in a typical 2D topological insulator $1 T^{\prime}$-TMD. Previous works showed that $\mathrm{MoTe}_{2}$ and $\mathrm{WTe}_{2}$ monolayers are semimetallic, rather than semiconducting [27]. Thus, in our study, we focus on disulfide and diselenide monolayers. Only diagonal components of $\mathcal{M}$ are considered (we will use $\mathcal{M}_{11}$ and $\mathcal{M}_{22}$ with Voigt notation where $11 \leftrightarrow 1111,22 \leftrightarrow 2222$ ), as off-diagonal effect is small and can be deduced mechanically. The geometric structure of $1 T^{\prime}$-TMD monolayer is shown in Fig. 1(a). We carefully optimize the lattice constants, thickness, and calculate elastic constants, and Poisson's ratio (see the Supplemental Material [28]). The mechanical properties of each $1 T^{\prime}$-TMD monolayer are nearly isotropic, with the largest 
(a)

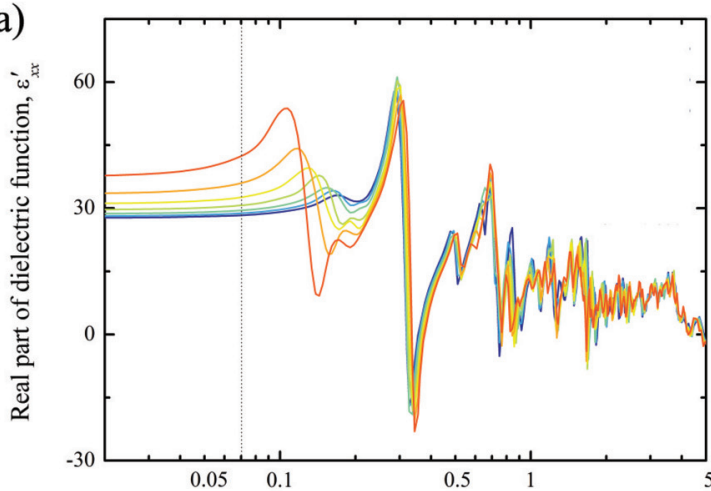

(c)

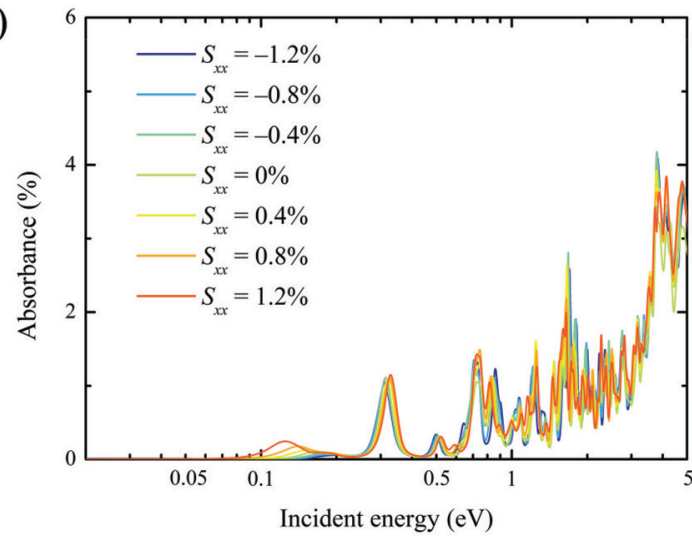

(b)

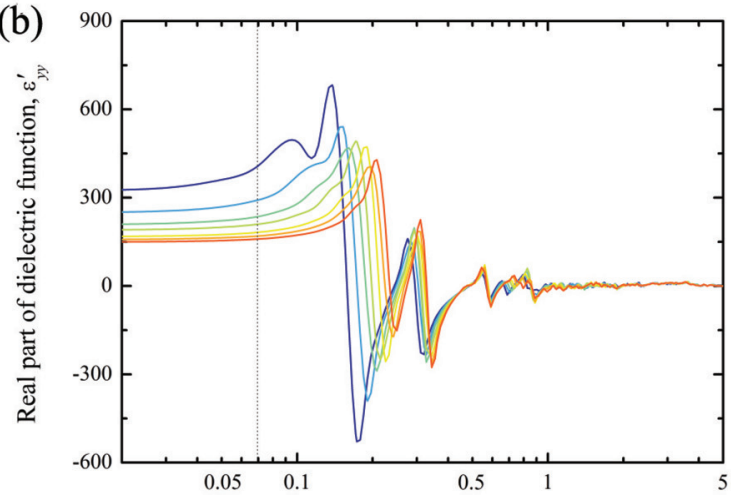

(d)

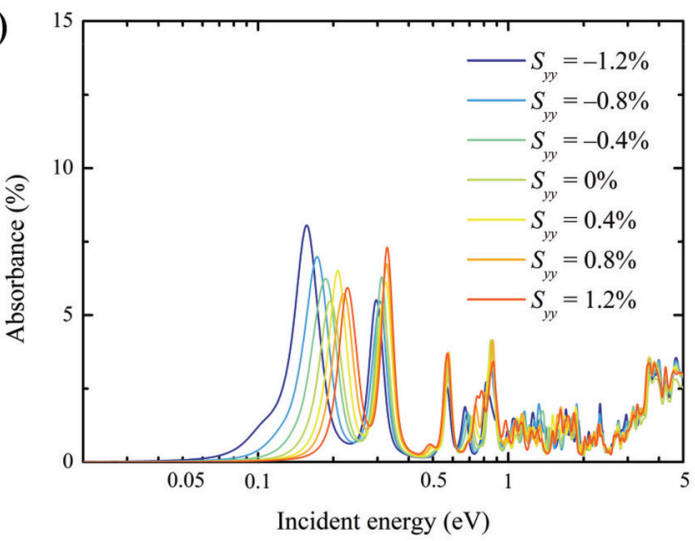

FIG. 2. Dielectric function of $1 T^{\prime}-\mathrm{MoS}_{2}$ monolayer under (a) $x$ - and (b) $y$-uniaxial strains, calculated by RPA. Corresponding absorbance spectra are shown in (c) and (d). Positive and negative strain values represent tensile and compressive strains, respectively. Poisson's ratio effects have been included in the simulation, e.g., for an $x$-direction uniaxial strain $S_{x x}$, a corresponding $S_{y y}=-v_{21} S_{x x}$ is also applied to mimic the stress-free condition in the $y$ direction.

difference within $10 \%$. Electronically speaking, if the spinorbit coupling (SOC) is not included, the valence band and conduction band touch each other and form Dirac points at $\pm \Lambda_{0}$ [Fig. 1(b)]. Finite band gap opens at these points once SOC is included. Typical electronic band structure of $1 T^{\prime}$ TMD is plotted in Fig. 1(c).

The two bands near Fermi level transform differently [Fig. 1(c)], hence, their optical selection behaviors are different, leading to anisotropic optical response. To quantify it, we calculate their dielectric function using random phase approximation (RPA) [45]. The second order optical response $\left(\chi^{(2)}\right)$ diminishes owing to the inversion symmetry. In the independent particle picture, the macroscopic dielectric function can be computed by [46]

$$
\overleftrightarrow{\boldsymbol{\varepsilon}}(\omega)=1-\frac{e^{2}}{2 \pi^{2}} \int d \mathbf{k} \sum_{c, v} \frac{\left\langle u_{v, \mathbf{k}}\left|\nabla_{\mathbf{k}}\right| u_{c, \mathbf{k}}\right\rangle\left\langle u_{c, \mathbf{k}}\left|\nabla_{\mathbf{k}}\right| u_{v, \mathbf{k}}\right\rangle}{E_{c, \mathbf{k}}-E_{v, \mathbf{k}}-\hbar \omega-i \xi},
$$

which could yield RPA response via solving Dyson's equation. Here $\left|u_{n, \mathbf{k}}\right\rangle$ and $E_{n, \mathbf{k}}$ are cell periodic part of Bloch wave function and its eigenvalue of band $n$ at $\mathbf{k}$. The denominator of Eq. (6) infers that low energy transitions dominate the optical response, and its numerator (interband Berry connection) evaluates the transition strength. In a band inverted topological insulator, both the valence and conduction band are mixed with same type of orbital components and the band gap is generally small. Owing to the symmetry selection, one anticipates large transition strength under the $y$-polarized light shining, while allowed transition for the $x$ polarization has small strength. This yields largely anisotropic dielectric functions, and can be verified in a $\boldsymbol{k} \cdot \boldsymbol{p}$ model [28].

We perform calculations of dielectric functions in a $3 \mathrm{D}$ supercell with vacuum space. In order to eliminate vacuum contribution, we follow previously suggested scheme, $\left(\varepsilon^{\mathrm{cal}}-1\right) d=\left(\varepsilon^{2 \mathrm{D}}-1\right) h[47,48]$, which is based on a parallel capacitor model [49]. The superscript "cal" and "2D" refers to supercell (with $z$-axis lattice constant $d$ ) calculated results and rescaled 2D (with thickness $h$ ) results, respectively. Figure 2 shows the dielectric functions and optical absorbance of $\mathrm{MoS}_{2}$ under different uniaxial strains. The absorbance is evaluated by $A_{i j}(\omega)=1-\exp \left(-\omega \varepsilon_{i j}^{\prime \prime} d / c_{0}\right)$, with $c_{0}$ the speed of light in vacuum. At the equilibrium state $\left(S_{x x}=S_{y y}=0\right)$, we observe clear and strong anisotropy between the $x$ and $y$ direction. The first absorption peak occurs at $0.17 \mathrm{eV}$ (with $A=0.1 \%$ ) and $0.19 \mathrm{eV}$ (with $A=5.4 \%$ ) for the $x$-polarized and $y$-polarized light, respectively. This indicates that $1 T^{\prime}$-TMDs are optically anisotropic. According to the Kramers-Kronig relationship, the real part of dielectric function below optical band gap is much larger for the $y$-polarized light than the $x$-polarized light, i.e., $\varepsilon_{x x}^{\prime} \ll \varepsilon_{y y}^{\prime}$. This is consistent with our above analysis.

Next, we consider uniaxial strain effects. Under artificial $x$-uniaxial compression, the band gap of $1 T^{\prime}-\mathrm{MoS}_{2}$ reduces. This makes the first peak in the absorbance spectrum shifts toward lower energy (red shift). Tension effect is opposite. Below the band gap (we take incident energy $\hbar \omega_{0}=0.07 \mathrm{eV}$ 

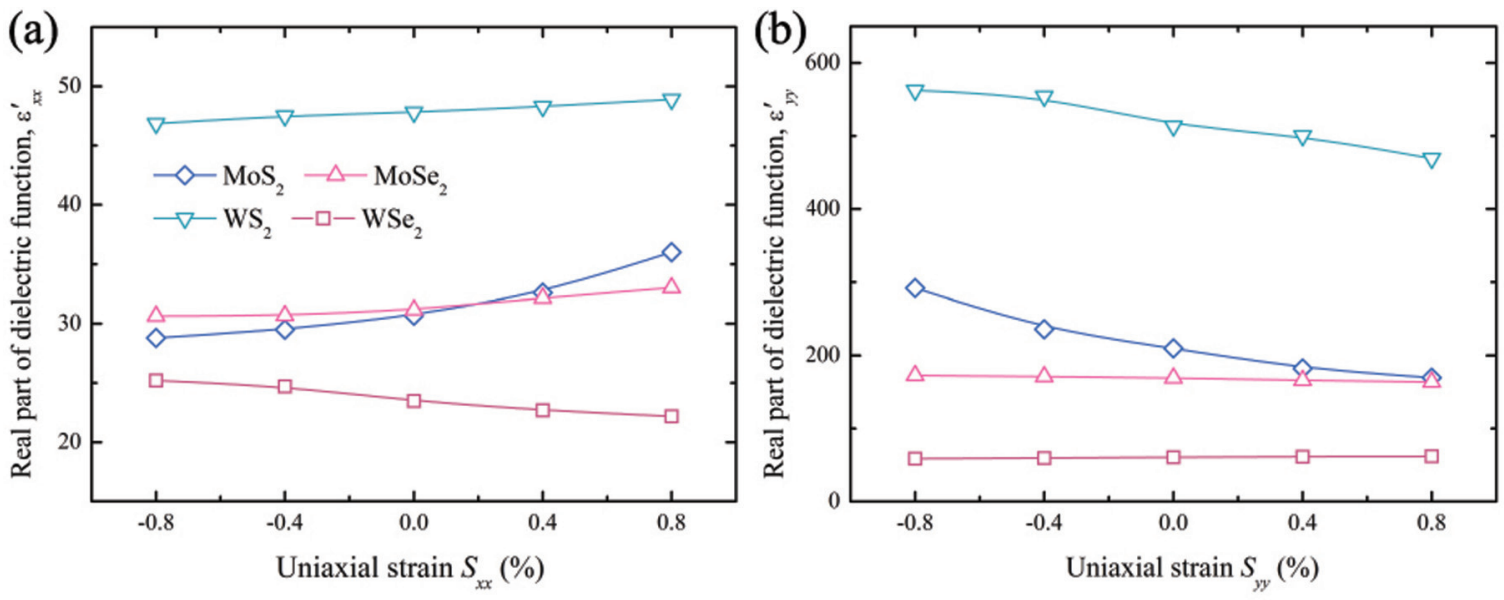

FIG. 3. Variation of real part of dielectric function at incident energy of $0.07 \mathrm{eV}$ under different uniaxial strains.

as an example, in mid-infrared region, dashed vertical line in Fig. 2), the real part of dielectric function $\varepsilon_{x x}^{\prime}\left(\omega_{0}\right)$ is 28.3 for $S_{x x}=-1.2 \%$ and 42.0 for $S_{x x}=1.2 \%$. Similarly, a $y$ uniaxial tensile strain reduces band gap and hence the first absorbance peak moves to the lower energy. The real part of dielectric function varies in accordance. Below the band gap, the $\varepsilon_{y y}^{\prime}\left(\omega_{0}\right)$ becomes 408.3 for $S_{y y}=-1.2 \%$ and 159.4 for $S_{y y}=1.2 \%$, which gives a much larger difference than that in the $x$-polarization case.

For other systems, similar results are obtained. Taking an incident energy of $\hbar \omega_{0}=0.07 \mathrm{eV}$ where direct optical absorption can be omitted, the dielectric function variations with respect to uniaxial strains are shown in Fig. 3. One clearly observes almost linear relationship between $\varepsilon_{x x}^{\prime}\left(\omega_{0}\right)$ and $S_{i i}$ ( $i=x$ or $1, y$ or 2 ). As in the elastic deformation region, the strain $S$ and stress $X$ nearly form linear dependence. One could yield a 2D optostriction constant

$$
\mathcal{M}_{i i}^{2 \mathrm{D}}=\frac{1}{2} \frac{\varepsilon_{0} \partial \varepsilon_{i i}^{\prime}\left(\omega_{0}\right)}{\partial X_{i i}}=\frac{1}{2} \frac{\varepsilon_{0} \partial \varepsilon_{i i}^{\prime}\left(\omega_{0}\right)}{C_{i i} \partial S_{i i}},
$$

which can be converted into a conventional 3D constant by $\mathcal{M}_{i i}^{3 \mathrm{D}}=h \mathcal{M}_{i i}^{2 \mathrm{D}}$. We summarize our calculated optostriction coefficients in Table I. Positive (negative) value of $\mathcal{M}$ indicates tensile (compressive) strain exerted on the TMD. We observe large magnitude of $\mathcal{M}_{22}$ along for $\mathrm{MoS}_{2}$ and $\mathrm{WS}_{2}$ monolayer.

We apply Eq. (4) to evaluate uniaxial strains under different intensity of linearly polarized laser [Fig. 4(a)]. The laser intensity is $I_{0}=c_{0} \varepsilon_{0} \mathcal{E}^{2} / 2$. One sees that an $x$-polarized

TABLE I. Calculated optostriction coefficients $\mathcal{M}$ and $\eta$. The $\mathcal{M}^{2 \mathrm{D}}$ is for $2 \mathrm{D}$ materials, in unit of $\mathrm{nm} / \mathrm{V}^{2}$, and $\mathcal{M}^{3 \mathrm{D}}=\mathcal{M}^{2 \mathrm{D}} \times h$ (in unit of $\mathrm{nm}^{2} / \mathrm{V}^{2}$ ) is a rescaled $3 \mathrm{D}$ value, to compare with conventional results in 3D materials. The $\eta$ values are in unit of $10^{-9} \mathrm{~m}^{2} / \mathrm{W}$.

\begin{tabular}{lrrrrrr}
\hline \hline & $\mathcal{M}_{11}^{2 \mathrm{D}}$ & $\mathcal{M}_{22}^{2 \mathrm{D}}$ & \multicolumn{1}{c}{$\mathcal{M}_{11}^{3 \mathrm{D}}$} & $\mathcal{M}_{22}^{3 \mathrm{D}}$ & \multicolumn{1}{c}{$\eta_{11}$} & \multicolumn{1}{c}{$\eta_{22}$} \\
\hline $\mathrm{MoS}_{2}$ & 0.030 & -0.482 & 0.021 & -0.351 & 1.16 & -26.36 \\
$\mathrm{MoSe}_{2}$ & 0.015 & -0.051 & -0.011 & -0.038 & 0.83 & -2.86 \\
$\mathrm{WS}_{2}$ & 0.008 & -0.380 & -0.006 & -0.272 & 0.45 & -20.34 \\
$\mathrm{WSe}_{2}$ & -0.013 & 0.014 & -0.010 & 0.010 & -0.75 & 0.75 \\
\hline \hline
\end{tabular}

laser of $2 \times 10^{9} \mathrm{~W} / \mathrm{cm}^{2}$ could generate $x$ strain of $0.03 \%$, $0.02 \%, 0.01 \%$, and $-0.02 \%$ for $\mathrm{MoS}_{2}, \mathrm{MoSe}_{2}, \mathrm{WS}_{2}$, and $\mathrm{WSe}_{2}$, respectively. Under the $y$-polarized laser, the corresponding strains are $-0.53 \%,-0.06 \%,-0.41 \%$, and $0.02 \%$, respectively. Inspired by photostriction process, we define and calculate a coefficient $\eta_{i i}=S_{i i} / I_{0}$, which measures strain under unit laser intensity (Table I). We see that $\mathrm{MoS}_{2}$ and
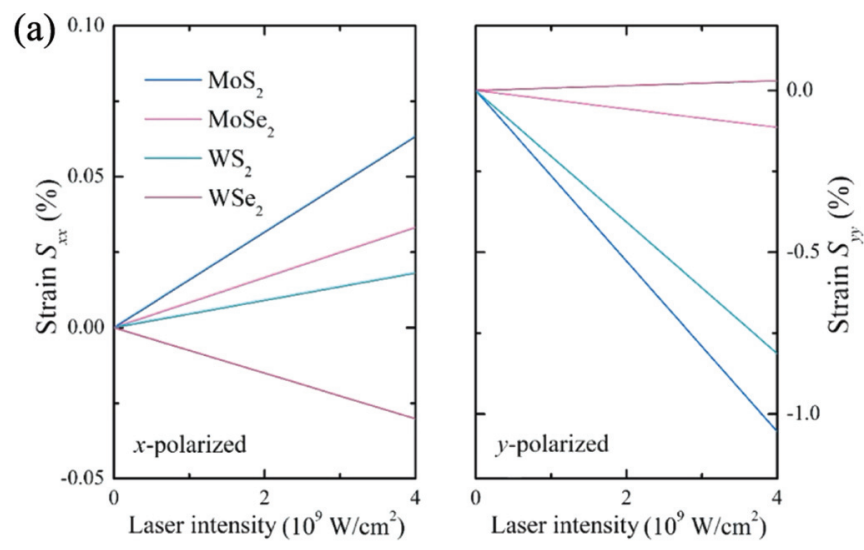

(b)

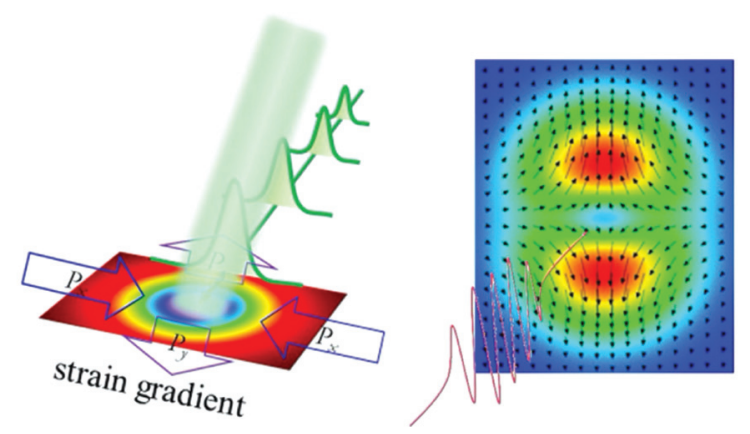

FIG. 4. (a) Variation of uniaxial strain (along $x$ and $y$ direction) with respect to laser intensity. (b) Schematics of a Gaussian beam laser triggered strain gradient (color scheme: blue to red indicates large to small local strains) with optoflexoelectric polarization (hollow arrows). Right panel shows 2D plot of strain gradient (colormap) and optoflexoelectric polarization texture (arrows). 

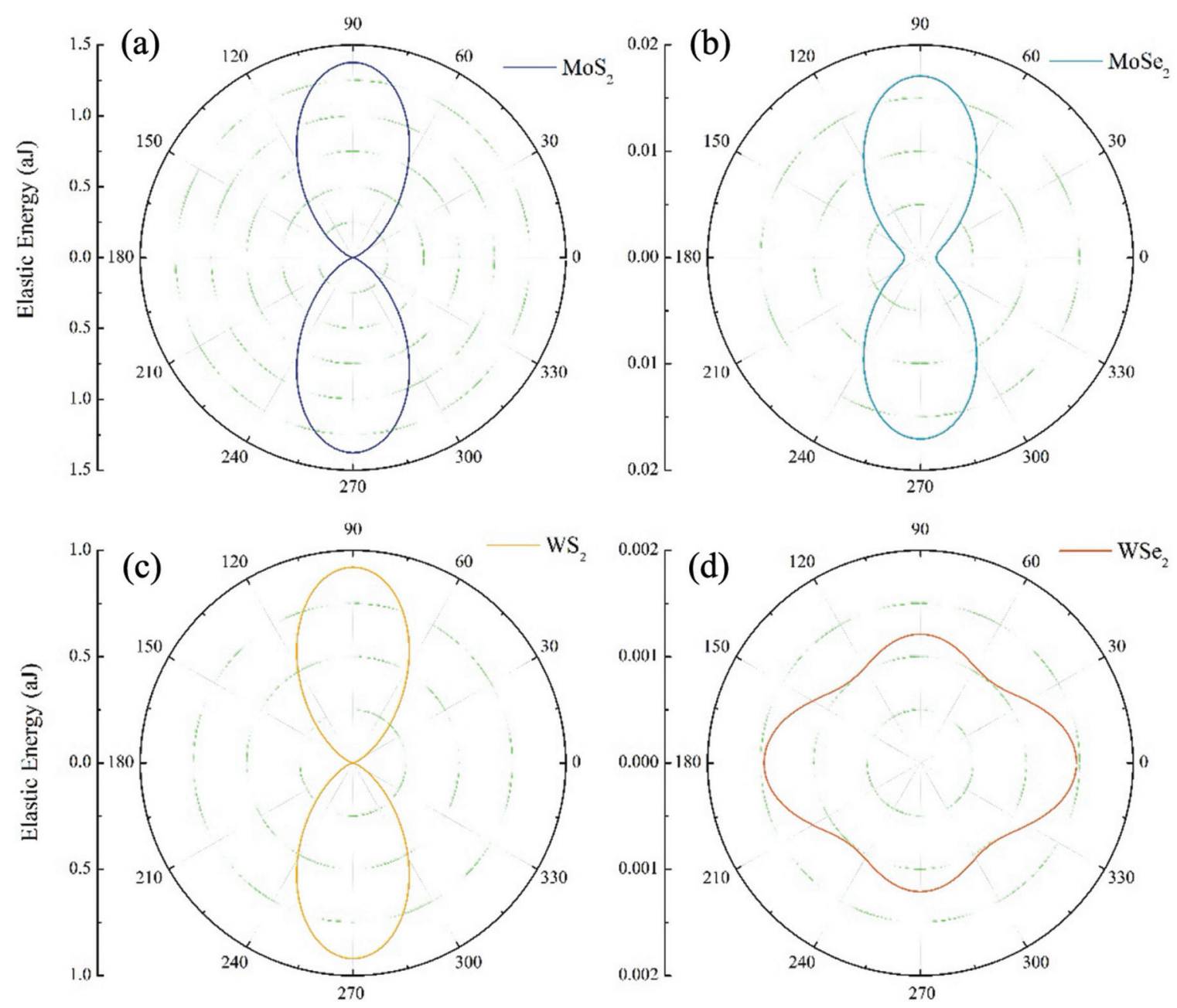

FIG. 5. Elastic strain energies (per unit cell) stored by linearly polarization laser $\left(I_{0}=2 \times 10^{9} \mathrm{~W} / \mathrm{cm}^{2}\right)$ with respect to its polarized direction. Strong anisotropy can be observed in (a) $\mathrm{MoS}_{2}$, (b) $\mathrm{MoSe}_{2}$, and (c) $\mathrm{WS}_{2}$, while the (d) $\mathrm{WSe}_{2}$ shows slight anisotropy.

$\mathrm{WS}_{2}$ has over $20 \times 10^{-9} \mathrm{~m}^{2} / \mathrm{W}$ magnitude of $\eta_{22}$, which is order of magnitude larger than the photostriction coefficients for nonpolar $\mathrm{Si}$ or Ge semiconductors [50-52], and typical perovskites such as $\mathrm{PbTiO}_{3}$ and $\mathrm{BiFeO}_{3}$ films [53,54].

The difference between $\mathcal{M}_{11}$ and $\mathcal{M}_{22}$ suggests a large in-plane anisotropic optostriction. Figure 5 shows elastic energy stored by illuminating a linearly polarized laser with an arbitrary polarization direction $[\hat{n}=(\cos \theta, \sin \theta)]$. The polar plot illustrates an approximate "8" shape curves. For example in $\mathrm{MoS}_{2}$, the largest elastic energy stored reaches 1.37 aJ per unit cell $(2.57 \mathrm{~kJ} 7 / \mathrm{g})$, while the smallest energy is $0.01 \mathrm{aJ}$ per unit cell $(0.02 \mathrm{~kJ} / \mathrm{g})$.

Unlike Maxwell stress, this optostriction depends on the sign of $\varepsilon_{i i}^{\prime}\left(\omega_{0}\right)$ variation under strain, which can be either tensile or compressive. Note that current elastic strain engineering in 2D materials usually applies external tensile strain. External compressive loading is not applicable due to the instability of out-of-plane acoustic mode. For example, according to the Euler-Bernoulli theory [55], the critical compressive $y$ strain of a TMD strut can be estimated by $\left|S_{y y}^{c r}\right|=\frac{\pi^{2}}{12} \frac{C_{22}}{C_{11}} \frac{h^{2}}{\left(K L_{y}\right)^{2}}$, where $L_{y}$ denotes its lateral length, and $K$ is pinning parameter ( $K=1$ in the simple supported case). Then a TMD monolayer with lateral length of $10 \mathrm{~nm}$ could sustain external compressive strain no more than $0.3 \%$ before mechanical instability. This is also seen in a recent experiment [56]. On the other hand, our predicted optostriction compression is intrinsic [28], which does not suffer from such instability.

We also propose that this laser inducing strain field is promising to generate large area inhomogeneous strain field $\left(\left|\nabla_{\mathbf{r}} \mathbf{S}\right| \neq 0\right)$ and realize detectable flexoelectric polarization, which is essential for novel electronic devices [57-60]. When a Gaussian beam laser is shining onto the sample [Fig. 4(b)], its center generates the largest magnitude of strain. This strain field decays towards its outside, with decaying rate depending on the Gaussian beam profile, $|\mathbf{S}| \propto e^{-2 r^{2} / w^{2}}$ (where $2 w$ is the full width of the beam at $1 / e^{2}$ and on the order of $10^{1}-10^{2} \mathrm{~nm}$ ). Hence, the laser could serve as a mesoscopic "artificial atom" to generate strain gradient field [61,62], avoiding direct contacts with the sample as in conventional nanoindentation method or possessing defects. Thus, rather than out-of-plane flexoelectricity which is usually measured by mechanically bending 2D materials, here in-plane flexoelectric polarization can be observed and measured. Using a simple model, we find that this polarization is proportional to strain gradient and its Born effective charge. The magnitude is on the order of $10^{-10} \mathrm{C} / \mathrm{m}$, comparable with typical intrinsic 
2D ferroelectrics, such as group-IV monochalcogenides [10]. As an inversion of elasto-optical effect [63], our predicted optostriction process does not require any symmetry constraints, in contrast to the electromechanical couplings in piezoelectrics.

In our model, due to the limitation of assigned 3D periodic boundary condition in the DFT framework, we use flat model at the zero temperature. One may wonder if thermal activation would induce a significant nonzero in-plane stress, as well as nonflat configuration. Now we make a brief estimate on thermally induced ripples and their in-plane effects for a nearly flat sheet. Suppose $h(\mathbf{x})$ is the thermally induced outof-plane displacement in real space, then its spectrum can be characterized by $\left\langle\bar{h}^{2}(\mathbf{q})\right\rangle=\frac{k_{B} T}{L^{2} \kappa q^{4}}[64,65]$, where $h(\mathbf{q})$ is the height in the Fourier space corresponding to wave number $\mathbf{q}\left[h(\mathbf{q})=\int d^{2} \mathbf{x} e^{-i \mathbf{q} \cdot \mathbf{x}} h(\mathbf{x}) / L^{2}\right], L$ is the lateral length of the sheet (assumed to be square), and $\kappa$ is the bending stiffness. According to Ref. [66], the resultant net in-plane strain can be characterized as

$$
S_{\mathrm{th}}=-\frac{1}{2}\left\langle(\nabla h)^{2}\right\rangle \sim-\frac{k_{B} T}{4 \pi \kappa} \ln \left(\frac{L}{a}\right),
$$

which is contractile (here $a$ is the lattice spacing). This contraction does not result in additional stresses but rather change the equilibrium spacing of the atoms. In other words, it will not change the "stress-free" configuration, but there is a nonzero strain. The sheet will buckle if additional compressive loads are applied onto this stress-free configuration. The optostriction effect is not an external load. If the sheet is not constrained, it will create a deformation without inducing any stresses-which is just a change in the stress-free configuration. If there is no geometric/mechanical restrictions, the material will "shrink" in certain directions, but not causing excessive stresses. This is similar as temperature cooling contracts the objects without causing instability. Therefore, we do not anticipate that optostriction will lead to buckling (intrinsic strain), unless the sheet is geometrically constrained or mechanically loaded in certain ways.

In addition, based on Eq. (8), we estimate that the thermal contraction is not prominent in our case. From previous calculations [67,68], the bending stiffness of TMDs is around $10 \mathrm{eV}$. Therefore, the strain induced by thermal activation under room temperature is very small (on the order of $0.01 \%$ if the lateral length of sheet is a few tens nanometers). Hence, for simplicity, we neglect the long wavelength thermal buckling effects (acoustic phonon modes), which are also under stressfree condition. Experimentally, one may use a prebuckled sheet to measure the optostriction [28]. The prebuckling could release the excessive stress and maintain the configuration in the stress-free state. If an optostrictive compression occurs, the buckling becomes less slack and the sheet gets flatter. This might aid us to observe and verify optostriction in experiments. Finite temperature effects on ionic vibrations (optical phonon modes) are evaluated [28], which yield similar results as the case of the DFT at zero temperature model.

Before closing, we would like to briefly compare the alternating electric field induced optostriction and electrostatic field induced electrostriction. The alternating field is time dependent which does not couple with $\mathbf{D}_{0}$, while the electrostatic field is zero frequency where $\mathbf{D}_{0}$ contributes largely. Physically speaking, optics oscillates the electron subsystem, and a static field displaces both the ions and electrons. Technically, their main differences are: (1) To apply a static electric field in experiments, one usually has to electrochemically pattern electrodes onto samples. Such contacting fabrication may degrade the material behaviors, and apply additional back-stress onto the sample to make the effect sluggish. Additional chemical dopants or adsorbates might cause unwanted impurities and disorders. The optical illumination scheme is noncontacting and noninvasive. The material could respond to the light quickly under illumination. (2) The static electric field direction is not easily manipulated, once the electrodes are patterned. The optical field direction, intensity, and frequency are more flexible. (3) The static electric field is usually uniform, while laser intensity gradient can be easily achieved. (4) In the optostriction, laser frequency $\omega$ is an additive tuning parameter, while the electrostriction is only its low frequency limit. (5) The optomechanical approach is less susceptible to lattice damage, but can provide considerable strains. (6) Optical illumination may generate electron-hole pairs, which complicates the process. On the other hand, static electric field usually does not suffer such difficulty (but external/internal carriers still exist). (7) Optical shining pattern is in a spatial resolution of a few hundred nanometers to a few micrometers, while current advanced electrochemical patterning can be as small as a few tens of nanometers.

In conclusion, we predict a novel and promising effectoptostriction. We illustrate a large in-plane anisotropic optostriction response in $1 T^{\prime}$-TMD monolayers. One could generate large intrinsic compressive strains without any outof-plane instabilities. A focused laser beam can generate a spatially inhomogeneous strain field, to measure in-plane flexoelectricity. Hence, the laser beam serves as a mesoscopic "artificial atom" without directly contacting the sample. Recently, it has been shown that terahertz laser irradiation could drive irreversible phase transformation from $2 H$ to $1 T^{\prime}$ in $\mathrm{MoTe}_{2}$ [69]. This result and setup can be used to verify our prediction. In order to realize large optostriction coefficients, topological materials with band inversions or soft materials with small elastic constants would be more preferable.

This work is supported by the National Natural Science Foundation of China under Grants No. 21903063, No. 11974270, and No. 11904350, and the Young Talent Startup Program of Xi' an Jiaotong University. The authors acknowledge useful discussions with Prof. Ju Li at MIT and Prof. Andrej Košmrlj at Princeton University.
[1] V. Sazonova, Y. Yaish, H. Üstünel, D. Roundy, T. A. Arias, and P. L. McEuen, Nature (London) 431, 284 (2004).
[2] D.-Y. Khang, H. Jiang, Y. Huang, and J. A. Rogers, Science 311, 208 (2006). 
[3] T. Ding, V. K. Valev, A. R. Salmon, C. J. Forman, S. K. Smoukov, O. A. Scherman, D. Frenkel, and J. J. Baumberg, Proc. Natl. Acad. Sci. USA 113, 5503 (2016).

[4] Y. Yue, Y. Norikane, R. Azumi, and E. Koyama, Nat. Commun. 9, 3234 (2018).

[5] M. T. Ghoneim, A. Kutbee, F. Ghodsi Nasseri, G. Bersuker, and M. M. Hussain, Appl. Phys. Lett. 104, 234104 (2014).

[6] R. Fei and L. Yang, Nano Lett. 14, 2884 (2014).

[7] J. Qiao, X. Kong, Z.-X. Hu, F. Yang, and W. Ji, Nat. Commun. 5, 4475 (2014).

[8] N. Mao, J. Tang, L. Xie, J. Wu, B. Han, J. Lin, S. Deng, W. Ji, H. Xu, K. Liu, L. Tong, and J. Zhang, J. Am. Chem. Soc. 138, 300 (2016).

[9] Z. Tian, C. Guo, M. Zhao, R. Li, and J. Xue, ACS Nano 11, 2219 (2017).

[10] R. Fei, W. Kang, and L. Yang, Phys. Rev. Lett. 117, 097601 (2016).

[11] M. Wu and X. C. Zeng, Nano Lett. 16, 3236 (2016).

[12] S. Huang, Y. Tatsumi, X. Ling, H. Guo, Z. Wang, G. Watson, A. A. Puretzky, D. B. Geohegan, J. Kong, J. Li, T. Yang, R. Saito, and M. S. Dresselhaus, ACS Nano 10, 8964 (2016).

[13] S. Yang, Y. Yang, M. Wu, C. Hu, W. Shen, Y. Gong, L. Huang, C. Jiang, Y. Zhang, and P. M. Ajayan, Adv. Funct. Mater. 28, 1707379 (2018).

[14] H. Cai, B. Chen, G. Wang, E. Soignard, A. Khosravi, M. Manca, X. Marie, L.-Y. Chang, B. Urbaszek, and S. Tongay, Adv. Mater. 29, 1605551 (2017).

[15] D. H. Keum, S. Cho, J. H. Kim, D.-H. Choe, H.-J. Sung, M. Kan, H. Kang, J.-Y. Hwang, S. Wng Kim, H. Yang, K. J. Chang, and Y. H. Lee, Nat. Phys. 11, 482 (2015).

[16] S. Song, D. H. Keum, S. Cho, D. Perello, Y. Kim, and Y. H. Lee, Nano Lett. 16, 188 (2016).

[17] Y. Wang, J. Xiao, H. Zhu, Y. Li, Y. Alsaid, K. Y. Fong, Y. Zhou, S. Wang, W. Shi, Y. Wang, A. Zettl, E. J. Reed, and X. Zhang, Nature (London) 550, 487 (2017).

[18] Y.-C. Lin, D. O. Dumcenco, Y.-S. Huang, and K. Suenaga, Nat. Nanotech. 9, 391 (2014).

[19] Y. Guo, D. Sun, B. Ouyang, A. Raja, J. Song, T. F. Heinz, and L. E. Brus, Nano Lett. 15, 5081 (2015).

[20] L. Liu, J. Wu, L. Wu, M. Ye, X. Liu, Q. Wang, S. Hou, P. Lu, L. Sun, J. Zheng, L. Xing, L. Gu, X. Jiang, L. Xie, and L. Jiao, Nat. Mater. 17, 1108 (2018).

[21] X. Xu, S. Chen, S. Liu, X. Cheng, W. Xu, P. Li, Y. Wan, S. Yang, W. Gong, K. Yuan, P. Gao, Y. Yu, and L. Dai, J. Am. Chem. Soc. 141, 2128 (2019).

[22] S. Wu, V. Fatemi, Q. D. Gibson, K. Watanabe, T. Taniguchi, R. J. Cava, and P. Jarillo-Herrero, Science 359, 76 (2018).

[23] Y. Shi, J. Kahn, B. Niu, Z. Fei, B. Sun, X. Cai, B. A. Francisco, D. Wu, Z.-X. Shen, X. Xu, D. H. Cobden, and Y.-T. Cui, Sci. Adv. 5, eaat8799 (2019).

[24] S. Tang, C. Zhang, D. Wong, Z. Pedramrazi, H.-Z. Tsai, C. Jia, B. Moritz, M. Claassen, H. Ryu, S. Kahn, J. Jiang, H. Yan, M. Hashimoto, D. Lu, R. G. Moore, C.-C. Hwang, C. Hwang, Z. Hussain, Y. Chen, M. M. Ugeda, Z. Liu, X. Xie, T. P. Devereaux, M. F. Crommie, S.-K. Mo, and Z.-X. Shen, Nat. Phys. 13, 683 (2017).

[25] Z. Fei, T. Palomaki, S. Wu, W. Zhao, X. Cai, B. Sun, P. Nguyen, J. Finney, X. Xu, and D. H. Cobden, Nat. Phys. 13, 677 (2017).

[26] W. Hou, A. Azizimanesh, A. Sewaket, T. Peña, C. Watson, M. Liu, H. Askari, and S. M. Wu, Nat. Nanotech. 14, 668 (2019).
[27] X. Qian, J. Liu, L. Fu, and J. Li, Science 346, 1344 (2014).

[28] See Supplemental Material at http://link.aps.org/supplemental/ 10.1103/PhysRevResearch.2.022059 for computational details and verification of computing model, which includes Refs. [29-33], light and phonon interaction analysis, finite temperature effect, anisotropic optostriction, anisotropic optical response under $\boldsymbol{k} \cdot \boldsymbol{p}$ model, linear response of polarizability under strains, hybrid functional calculations on $\mathrm{MoS}_{2}$, and anisotropic in-plane optoflexoelectricity.

[29] G. Kresse and J. Furthmüller, Phys. Rev. B 54, 11169 (1996).

[30] J. P. Perdew, K. Burke, and M. Ernzerhof, Phys. Rev. Lett. 77, 3865 (1996).

[31] J. P. Perdew, A. Ruzsinszky, G. I. Csonka, O. A. Vydrov, G. E. Scuseria, L. A. Constantin, X. Zhou, and K. Burke, Phys. Rev. Lett. 100, 136406 (2008).

[32] P. E. Blöchl, Phys. Rev. B 50, 17953 (1994).

[33] H. J. Monkhorst and J. D. Pack, Phys. Rev. B 13, 5188 (1976).

[34] J. Heyd, G. E. Scuseria, and M. Ernzerhof, J. Chem. Phys. 118, 8207 (2003).

[35] J. Heyd, G. E. Scuseria, and M. Ernzerhof, J. Chem. Phys. 124, 219906 (2006).

[36] M. Stengel, N. A. Spaldin, and D. Vanderbilt, Nat. Phys. 5, 304 (2009).

[37] M. Stengel, D. Vanderbilt, and N. A. Spaldin, Phys. Rev. B 80, 224110 (2009).

[38] J. F. Nye, Physical Properties of Crystals: Their Representation by Tensors and Matrices (Oxford University Press, London, 1985).

[39] C. Paillard, B. Xu, B. Dkhil, G. Geneste, and L. Bellaiche, Phys. Rev. Lett. 116, 247401 (2016).

[40] R. Haleoot, C. Paillard, T. P. Kaloni, M. Mehboudi, B. Xu, L. Bellaiche, and S. Barraza-Lopez, Phys. Rev. Lett. 118, 227401 (2017).

[41] Y. Zhou, L. You, S. Wang, Z. Ku, H. Fan, D. Schmidt, A. Rusydi, L. Chang, L. Wang, P. Ren, L. Chen, G. Yuan, L. Chen, and J. Wang, Nat. Commun. 7, 11193 (2017).

[42] B. Kundys, M. Viret, D. Colson, and D. O. Kundys, Nat. Mater. 9, 803 (2010).

[43] H. Hu, M. Liu, Z. F. Wang, J. Zhu, D. Wu, H. Ding, Z. Liu, and F. Liu, Phys. Rev. Lett. 109, 055501 (2012).

[44] H. Hu, H. Ding, and F. Liu, Sci. Rep. 5, 8212 (2015).

[45] M. Gajdoš, K. Hummer, G. Kresse, J. Furthmüller, and F. Bechstedt, Phys. Rev. B 73, 045112 (2006).

[46] K. W.-K. Shung, Phys. Rev. B 34, 979 (1986).

[47] Z. Jiang, Z. Liu, Y. Li, and W. Duan, Phys. Rev. Lett. 118, 266401 (2017).

[48] J. Zhou, H. Xu, Y. Li, R. Jaramillo, and J. Li, Nano Lett. 18, 7794 (2018).

[49] A. Laturia, M. L. Van de Put, and W. G. Vandenberghe, npj 2D Mater. Appl. 2, 6 (2016).

[50] C.-Y. Peng, C.-F. Huang, Y.-C. Fu, Y.-H. Yang, C.-Y. Lai, S.-T. Chang, and C. W. Liu, J. Appl. Phys. 105, 083537 (2009).

[51] J. R. Buschert and R. Colella, Solid State Commun. 80, 419 (1991).

[52] T. Figielski, Phys. Status Solidi 1, 306 (1961).

[53] D. Daranciang et al., Phys. Rev. Lett. 108, 087601 (2012).

[54] D. Schick, M. Herzog, H. Wen, P. Chen, C. Adamo, P. Gaal, D. G. Schlom, P. G. Evans, Y. Li, and M. Bargheer, Phys. Rev. Lett. 112, 097602 (2014). 
[55] D. W. A. Rees, Mechanics of Optimal Structural Design: Minimum Weight Structures (John Wiley \& Sons, New York, 2009).

[56] H. Ren, Z. Xiang, E. Wang, Z. Yuan, Y. Sun, K. Zhu, B. Wang, X. Wang, H. Ding, P. Liu, L. Zhang, J. Wu, S. Fan, X. Li, and K. Liu, ACS Nano 13, 3106 (2009).

[57] A. Abdollahi, F. Vásquez-Sancho, and G. Catalan, Phys. Rev. Lett. 121, 205502 (2018).

[58] W. Shi, Y. Guo, Z. Zhang, and W. Guo, J. Phys. Chem. Lett. 9, 6841 (2018).

[59] T. D. Nguyen, S. Mao, Y.-W. Yeh, P. K. Purohit, and M. C. McAlpine, Adv. Mater. 25, 946 (2013).

[60] S. Mao and P. K. Purohit, J. Appl. Mech. 81, 081004 (2014).

[61] J. Feng, X. Qian, C.-W. Huang, and J. Li, Nat. Photon. 6, 866 (2012).

[62] J. Li, K. J. van Vliet, T. Zhu, S. Yip, and S. Suresh, Nature (London) 418, 307 (2002).
[63] L. Chen, Y. Yang, Z. Gui, D. Sando, M. Bibes, X. K. Meng, and L. Bellaiche, Phys. Rev. Lett. 115, 267602 (2015).

[64] D. R. Nelson, T. Piran, and S. Weinberg, Statistical Mechanics of Membranes and Surfaces, 2nd ed. (World Scientific, Singapore, 2004).

[65] A. Košmrlj and D. R. Nelson, Phys. Rev. E 88, 012136 (2013).

[66] F. Ahmadpoor P. Wang, R. Huang, and P. Sharma, J. Mech. Phys. Solids 107, 294 (2017).

[67] K. Lai, W.-B. Zhang, F. Zhou, F. Zeng, and B.-Y. Tang, J. Phys. D: Appl. Phys. 49, 185301 (2016).

[68] N. K. Nepal, L. Yu, Q. Yan, and A. Ruzsinszky, Phys. Rev. Mater. 3, 073601 (2019).

[69] J. Shi, Y.-Q. Bie, W. Chen, S. Fang, J. Han, Z. Cao, T. Taniguchi, K. Watanabe, V. Bulović, E. Kaxiras, P. Jarillo-Herrero, and K. A. Nelson, arXiv:1910.13609. 